\title{
LA EXPERIENCIA DEL NOSOTROS EN EL SER Y LA NADA. SARTRE EN EL UMBRAL DE LO SOCIAL
}

\author{
THE EXPERIENCE OF THE WE/US IN BEING AND NOTHINGNESS. SARTRE AT THE \\ THRESHOLD OF THE SOCIAL
}

\author{
Daniel Alvaro* \\ Consejo Nacional de Investigaciones Científica y Técnicas / Universidad de Buenos Aires-IIGG, \\ Buenos Aires - Argentina.
}

Recibido noviembre de 2017/Received November, 2017

Aceptado enero de 2018/Accepted January, 2018

\section{RESUMEN}

El presente artículo se interroga por el lugar que ocupa lo social al interior de la teoría sobre la existencia humana que Sartre desarrolla en El ser y la nada: Ensayo de ontología fenomenológica (1943). Con ese fin se analizan paso a paso los principales argumentos y conceptos sartreanos referidos a la existencia del otro, la relación fundamental con el otro, las relaciones concretas con el otro y, finalmente, la experiencia del nosotros.

Palabras Clave: Nosotros, Ontología, Relación, Existencia, Sartre.

\begin{abstract}
The present article questions the place that the social occupies within the theory of the human existence, developed by Sartre in Being and Nothingness: An Essay on Phenomenological Ontology (1943). To that end, it will analyse systematically the main Sartrean arguments and concepts referred to the existence of the other, the fundamental relationship with the other, the concrete relationships with the other and, finally, the experience of the We/Us.
\end{abstract}

Key Words: We/US, Ontology, Relationship, Existence, Sartre.

La publicación de El ser y la nada (1943) es recordada como un acontecimiento que modificó sensiblemente el escenario intelectual y cultural de la época. A pesar de la considerable extensión del libro, la densidad del razonamiento que sirve de hilo conductor y el lenguaje técnico en el que está escrito, con el correr de los años su alcance excedió ampliamente el campo académico al que parecía estar destinado. Se trata de la primera gran obra filosófica de Sartre y sin duda la más famosa. Allí desarrolla algunas de las tesis fundamentales de lo que se dio en llamar "existencialismo" etiqueta que Sartre en un comienzo rechazó pero que finalmente terminó por aceptar-, basadas en la idea de libertad y la plena responsabilidad del hombre respecto de su existencia. Desde su

* Autor correspondiente / Correspondig author: danielalvaro@ gmail.com 
aparición, y más aún desde el final de la guerra, el libro provocó reacciones apasionadas de aprobación y rechazo, tal como sucedió a una escala todavía mayor con las novelas y piezas dramáticas que el autor publicó en ese mismo período. Si bien el existencialismo atraía fervientes seguidores, especialmente entre los jóvenes, al mismo tiempo ganaba enemigos entre los defensores de la religión, la moral y las buenas costumbres, que según la opinión corriente, la moda existencialista venía a poner en cuestión. Desde un punto de vista teórico, las reacciones no fueron menos tajantes. Por una parte, entre los próximos a Sartre se encontraba, además de un reducido círculo de discípulos, el grupo de colegas con los que fundó la revista Les Temps Modernes en 1945 (Raymond Aron, Simone de Beauvoir, Michel Leiris, Maurice Merleau-Ponty, Albert Ollivier \& Jean Paulhan). Por otra parte, entre sus principales detractores figuraban, además de los críticos religiosos, algunos intelectuales vinculados al Partido Comunista Francés. En términos generales, estos últimos le reprochaban ser el portavoz de una corriente de pensamiento subjetivista o individualista, inclinada a la inacción, contemplativa, y pesimista acerca del porvenir de la humanidad. En síntesis, lo acusaban de ser el referente de una nueva filosofía burguesa.

Cabe recordar que Sartre fue sensible a estas críticas, no solo por la violencia con que muchas de ellas fueron lanzadas, sino también porque estas se agudizaron en el momento mismo en que aquel comenzaba a involucrarse más activamente en las luchas sociales y políticas de la época. Sartre contestó a estas críticas en varias ocasiones y de distintas maneras. La famosa conferencia, El existencialismo es un humanismo (1946), fue una de las primeras respuestas a las objeciones que suscitó la publicación de El ser y la nada ${ }^{1}$. Desde entonces, el progresivo acercamiento de Sartre al marxismo hizo que sus réplicas variaran significativamente a lo largo del tiempo. Su segunda gran obra filosófica, Crítica de la Razón dialéctica (cuyo primer tomo apareció en 1960, y el segundo, póstumamente, en 1985), puede leerse como una explicación concluyente y hasta cierto punto concesiva con las tempranas críticas marxistas que pusieron de relieve la infravaloración del materialismo y de las determinaciones objetivas que pesan sobre las relaciones humanas en el curso de la historia ${ }^{2}$.

Entre las críticas que le hicieron los comunistas en la década de 1940, quisiera indagar aquella que le recrimina una reflexión insuficiente de la dimensión social e histórica del hombre. El mismo hecho de que la crítica comunista condene la insuficiencia de su reflexión en este aspecto supone que, al menos en cierta medida, existe en este libro un intento por pensar la vida colectiva. Lo que me interesa, puntualmente, es interrogar el lugar de lo social en la tesis sobre la existencia humana que Sartre pone a prueba en El ser y la nada.

Al margen de cualquier valoración, el intento es indiscutible. Es verdad que, comparativamente, este ocupa un lugar reducido en el texto pero no por eso es menos sugestivo y rico en ideas. Las nociones y proposiciones que refieren más directamente a la vida en común forman parte de lo que Sartre llama la "experiencia del nosotros". A fin de comprender el significado de esta experiencia, el presente artículo propone un análisis en varios pasos y en distintos niveles que será desarrollado en las cuatro secciones siguientes. En la primera de ellas examino el estatuto de la ontología fenomenológica sartreana a partir de una cuestión central y dilemática para la teoría social como es el "punto de partida" o fundamento. La pregunta que intento contestar allí es si es posible deducir de este pensamiento sobre el ser algo así como una ontología social. En la segunda sección me centro en la cuestión del otro. Tomo en consideración la teoría de Sartre sobre la existencia del otro y sobre la relación original entre las conciencias. Finalmente, en las secciones tercera y cuarta abordo el tema de las relaciones concretas con el otro, así como distintos aspectos de la denominada "experiencia del nosotros"3.

\section{1. ¿Ontología social?}

En un pasaje muy citado de El ser y la nada leemos: "Mi proyecto último e inicial — pues es los dos a la vez- siempre es, lo veremos, el bosquejo de una solución al problema del ser. Pero esta solución no es primero concebida y después realizada: nosotros somos esta solución, la hacemos existir por nuestro compromiso mismo y por lo tanto no podríamos captarla más que viviéndola" (2007, p. 507). En pocas y concentradas palabras, Sartre define su proyecto general y al mismo tiempo establece uno de los principios de la filosofía existencialista que desde entonces permanecerá asociada a su nombre. Si se toma en cuenta su inmensa producción, resulta claro que el proyecto 
que consiste en esbozar "una solución al problema del ser" aplica muy especialmente al momento de la concepción de El ser y la nada. El libro se define desde el subtítulo como un Ensayo de ontología fenomenológica. El trabajo emprendido por Sartre en su obra capital parte a la búsqueda del sentido original del ser. Esto no significa que esta búsqueda haya encontrado allí un principio ni un fin. Se trata de una investigación de largo aliento que puede rastrearse en publicaciones anteriores y posteriores a esta. Aunque en lo concerniente a la "elucidación del sentido del ser" no se encontrará en su producción una fuerza y una determinación semejantes a las que llega el autor en este libro.

Allí Sartre intenta responder a la pregunta por el "ser en general", comprendidas las distintas regiones que este abarca, y afirma dar con la respuesta. El problema encuentra una solución en la existencia del hombre. Si bien no se interroga únicamente por el ser del hombre, la pregunta por el ser en general, y en consecuencia por los distintas tipos de ser, solo cobra sentido al interior de un universo humano. De ahí, pues, que ante el planteo del problema ontológico por excelencia Sartre pueda afirmar que nosotros somos la solución. El pasaje en cuestión no hace más que afirmar a su manera un principio axial del existencialismo, a saber, que "la existencia precede a la esencia". Esto significa que la definición de la esencia del hombre no solo es posterior sino que está condicionada por su existencia, lo que desde la visión sartreana equivale a decir, por su libertad. Se entiende, pues, que la frase tomada de El ser y la nada indica algo de este orden: la solución al problema del ser no puede ser concebida y luego realizada por el simple hecho de que no está dada; nosotros mismos, en nuestra experiencia vital, somos la respuesta a la pregunta. Si esta guarda algún sentido hay que buscarlo en la existencia, vale decir en nuestros actos, no en una supuesta naturaleza humana anterior de la cual aquella dependería. Ahora bien, lo que está lejos de ser evidente en dicha frase es el significado del "nosotros" (nous) allí comprometido. La construcción gramatical de este enunciado - como el de tantos otros que pueblan el libro y que por lo demás se le parecen-induce a un equívoco que Sartre no supo o no pudo evitar: el equívoco entre una respuesta individual y una respuesta colectiva a la pregunta por el ser.

¿Qué quiere decir, en términos ontológicos, que "nosotros somos la solución" al "problema del ser"? ¿Acaso significa que la pregunta que interroga el ser desemboca en una respuesta que afirma el sentido primigenio de la existencia colectiva? ¿Somos nosotros, seres humanos en situación los unos con respecto a los otros, el fundamento ontológico de la existencia? Dicho sin rodeos: ¿supone esta fórmula directa o indirectamente una ontología social o de lo social?

Si bien este uso del pronombre personal "nosotros" puede conducir legítimamente a un razonamiento de este tipo, es preciso aclarar de entrada cualquier confusión al respecto. Sobre todo si se tiene en cuenta que la argumentación de Sartre no deja lugar a duda en este sentido. Tal como lo explicita una y otra vez a lo largo del ensayo, su ontología fenomenológica parte del cogito cartesiano o, para decirlo en sus propios términos, de la subjetividad del individuo. "Un estudio de la realidad-humana debe comenzar por el cogito" (2007, p. 121). Desde esta perspectiva, el "pienso, luego existo" de Descartes es el único punto de partida verdadero, el único del que no se puede dudar, y por lo tanto un fundamento seguro para estudiar la realidad del hombre en sus diferentes dimensiones.

(...) el cogito cartesiano no hace más que afirmar la verdad absoluta de un hecho: el de mi existencia; asimismo, el cogito un poco ampliado del que nos servimos aquí revela como un hecho la existencia del otro y mi existencia para otro ${ }^{4}$. (2007, p. 322).

A partir de estas afirmaciones preliminares no es posible sacar ninguna conclusión acerca del modo en que Sartre piensa la existencia colectiva, que en el fondo es la cuestión que me interesa tratar aquí, pero al menos pueden servir para mostrar que la existencia individual es el fundamento que sirve de base a la ontología expuesta en El ser y la nada ${ }^{5}$.

Al escribir "nosotros", Sartre habla en nombre de todos los hombres y, por extensión, de todas las mujeres. Dicho de otra forma, Sartre habla en nombre de la humanidad. En todo caso, lo que importa subrayar es que la experiencia a la que se refiere es la de cada sujeto individual, no la experiencia de un conjunto, ni tampoco la de la totalidad de los sujetos. Se trata de lo que la teoría de la enunciación denomina un "nosotros inclusivo", aquel en el cual todos estamos involucrados. 
Pero este involucramiento, al que efectivamente ninguna existencia escapa, afecta a todos porque en principio afecta a cada uno. Por consiguiente, para decirlo con términos de Durkheim, el nosotros en cuestión es un fenómeno "general", no un fenómeno "colectivo".

Decía más arriba que Sartre no supo o no pudo evitar la doble interpretación a la que se presta esta y otras frases semejantes. En cualquier caso, no es algo que se le haya pasado por alto. Precisamente, el último apartado del Capítulo III de la Tercera parte de El ser y la nada — apartado sobre el que volveré en la tercera sección de este artículo- comienza con una reflexión sobre el uso del pronombre "nosotros". Allí escribe: "es verdad que, frecuentemente, decimos 'nosotros'. La existencia misma y el uso de esta forma gramatical reenvían necesariamente a una experiencia real del Mitsein" (2007, p. 453). De este modo reconoce que la simple enunciación de la forma "nosotros" da cuenta de una experiencia efectiva, y por cierto cotidiana, que es la experiencia de "sercon" (Mitsein) otros. Pero agrega de inmediato: “"Nosotros' puede ser sujeto y, bajo esta forma, es asimilable a un plural del 'yo'. Y, ciertamente, el paralelismo de la gramática y el pensamiento es, en muchos casos, más que dudoso" (2007, p. 453). Este punto es de la mayor importancia para toda su argumentación sobre la relación con el otro, pues deja en claro que su duda, y más precisamente su objeción, no recae sobre la "experiencia del nosotros" en cuanto tal, sino más bien sobre el hecho de considerar el "nosotros" como un punto de partida verdadero, esto es, indudable, tal como lo es el “yo". En el mismo apartado, algunas líneas más abajo, afirma que mediante su crítica al concepto heideggeriano de "ser-con" — crítica que desarrolla en el Capítulo I de la Tercera parte, justo antes de exponer su propia explicación de la existencia del otro y de la relación con el otro- no busca "poner en duda la experiencia del nosotros". Su cometido, aclara, es "mostrar que esta experiencia no podía ser el fundamento de nuestra conciencia del otro", que aquella "no podría constituir una estructura ontológica de la realidad-humana" (2007, p. 454). Para Sartre, en suma: “(...) está claro que el nosotros no es una conciencia intersubjetiva, ni un ser nuevo que supera y engloba sus partes como un todo sintético, a la manera de la conciencia colectiva de los sociólogos" (2007, p. 454).
Tal vez ahora se vea con mayor claridad lo que el "nosotros" no es. Sin embargo, no podemos contentarnos con una caracterización exclusivamente negativa del fenómeno. En la medida en que se trata de una experiencia real, una experiencia que de una u otra forma termina por remitir siempre a una situación colectiva, será cuestión de interpretar, de manera positiva, en qué consiste este fenómeno. Con vistas a ello, primero hay que examinar una de las modalidades fundamentales del ser expuestas por Sartre: el "ser-para-otro" (être-pour-autrui) ${ }^{6}$. Y esto no solo por fidelidad al argumento y a la disposición misma de la densa trama conceptual de El ser y la nada, sino también, y ante todo, porque la experiencia del nosotros mantiene una relación de dependencia respecto de esta modalidad ontológica.

\section{La cuestión del otro}

El "ser-para-otro" es una de los tres tipos de ser a las que se hace referencia en el ensayo de ontología fenomenológica. Sartre le dedica la Tercera parte del libro. La Introducción y las dos primeras partes están consagradas al "ser-en-sí", al "ser-para-sî", y a los vínculos que existen entre ambos. A partir de la descripción de estos tres tipos de ser y de la explicación de sus múltiples relaciones el autor espera dar inicio a una "teoría metafísica del ser en general” (2007, p. 401). Tal es la meta que se propone alcanzar.

Aunque Sartre afirme que el ser-para-otro es tan fundamental como el ser-en-sí y el ser-para-sí, no se puede pasar por alto el hecho de que aquel ocupa el tercer lugar en el orden de exposición. A los efectos de un análisis que se proponga decir algo sobre la dimensión social de este pensamiento, resulta significativo que la estructura ontológica más directamente relacionada con la existencia del otro sea tratada en último lugar. El primer tercio del libro, donde "el otro" aparece ocasionalmente y siempre de manera accesoria al argumento central, está dedicado a la descripción y distinción del "en-sí" o ser de los fenómenos y el "para-sî" o ser de la conciencia. Estas son dos modalidades de ser opuestas y hasta incomunicadas entre sí, aunque se mantienen unidas por distintas clases de relaciones. Para decirlo abruptamente, la irrupción del otro como problema tiene lugar una vez que fue abordada la relación fundamental entre la conciencia y los fenómenos, esto es, entre el hombre y el mundo. Este particular ordenamiento 
no tiene nada de fortuito. Por el contrario, guarda absoluta coherencia con el punto de partida privilegiado y tiene, como veremos, consecuencias notables en la concepción sartreana del otro.

Sartre le atribuye a la "realidad-humana" traducción o adaptación del Dasein de Heideggerdos modos de existencia que, según afirma, se encuentran en el mismo nivel desde un punto de vista ontológico: el ser-para-sí y el ser-para-otro. "(...) la realidad-humana debe ser en su ser, en un único y mismo surgimiento, para-sí-para-otro" $\left(2007\right.$, p. 255) ${ }^{7}$. Es decir que, aunque heterogéneos, estos modos de existencia pertenecen a $m i$ ser. Son míos. La diferencia radica en que el "para-sí" se realiza por medio de la reflexión, mientras que el "para-otro" se realiza "delante de alguien", de algún otro o alguna otra. Sartre explica esta diferencia tomando como ejemplo el sentimiento de vergüenza que cualquiera puede experimentar cuando es visto por alguien haciendo un gesto torpe o vulgar. Y afirma que, en general, se siente vergüenza frente a otro, no como resultado de una actitud reflexiva de la conciencia. Así, el otro es definido en primer lugar como "mediador indispensable entre yo y yo mismo: tengo vergüenza de mí tal como me aparezco al otro" (2007, p. 260).

Ante todo, el otro aparece como aquel que me revela lo que soy. El otro me constituye en cuanto ser-para-otro sin que yo deje de ser, a la vez, ser-para-sí. El ser-para-otro — vale la pena insistir - es uno de los modos de existencia de la realidad-humana. No hay que confundirlo con el Otro. La existencia del otro es un problema en sí mismo, que conduce a Sartre a una larga disquisición sobre lo que él denomina "el escollo del solipsismo".

Si se deja de lado la posición solipsista más radical, aquella que afirma la "soledad ontológica", es decir, "que nada existe fuera de mí", por considerarla una mera especulación metafísica sin relación con la experiencia, queda el solipsismo moderado, aquel que afirma la existencia del otro apelando al sentido común, pero que es impotente para justificarla filosóficamente. Para Sartre, tanto la posición idealista como la realista, directamente en un caso e indirectamente en el otro, terminan por recaer en una actitud solipsista. Idealistas y realistas están convencidos de la existencia del otro, del "yo que no es yo", pero ni los unos ni los otros pueden evitar contradicciones profundas en su acercamiento a la problemática de la alteridad. El origen de estas contradicciones se encuentra en una presuposición compartida por ambas doctrinas: el presupuesto según el cual la "negación constituyente es negación de exterioridad" (2007, p. 269). De acuerdo con esto, entre "yo" y el "otro" existe una separación dada o natural. Desde un punto de vista empírico, mi cuerpo se relaciona con el cuerpo del otro exteriormente, según la relación espacial que rige para la totalidad de las cosas extensas. Pero lo mismo vale para las conciencias, cuya separación también se concibe espacialmente. Por caminos diversos, los realistas y los idealistas llegan a la misma conclusión: "es un espacio real o ideal el que nos separa del otro" (2007, p. 270).

De este modo, la relación con el otro es concebida bajo la forma de una pura exterioridad indiferenciada. Lo cual implica, como mínimo, desinteresarse de la afectación y determinación recíprocas entre mi ser y el ser del otro. Contra esta concepción, Sartre propone pensar la relación original con el otro como una "negación de interioridad". Pero antes de meterse de lleno en la elaboración de su propio planteamiento, evalúa uno a uno los argumentos sobre la existencia del otro desarrollados por Husserl, Hegel y Heidegger. Tres pensadores que, junto a Descartes, se encuentran entre los que más cuentan para él, al menos en el momento de la redacción de El ser y la nada. Para decirlo rápidamente, y por lo tanto sin poder hacer justicia a la crítica diferenciada que se lleva a cabo en el texto, Sartre reconoce en las filosofías de estos autores el intento por superar "el postulado de la negación externa", sin dejar de reprocharles el haber conservado intacta la consecuencia que acarrea tal postulado, a saber, "la afirmación de que mi vínculo fundamental con el otro es realizado a través del conocimiento" (2007, p. 271). A decir verdad, esta objeción está dirigida fundamentalmente a Husserl y Hegel. Ambos son cuestionados por considerar el ser del otro a título de objeto, lo que equivale a abordar el problema ontológico de la existencia del otro en términos de conocimiento. La objeción a Heidegger es ciertamente de otra naturaleza. Volveré sobre ella en la próxima sección.

A partir de estas críticas quedan establecidas "las condiciones necesarias y suficientes para que una teoría de la existencia del otro sea válida" (2007, p. 289). En primer lugar, el otro no es una simple probabilidad; es una certeza en correspondencia con el hecho de que hay un cogito con el cual se 
vincula. En segundo lugar, hay que partir del cogito cartesiano, vale decir, de "mi propio cogito", para que me descubra la existencia concreta y certera del otro. En tercer lugar, la existencia del otro no es, en principio, un objeto. En cuarto y último lugar, el otro se revela al cogito como no siendo yo. Esta negación es "interna", por oposición a la "externa", y se concibe como relación entre dos términos que se constituyen a partir de su mutua negación. Es una "relación negativa", "recíproca y de doble interioridad" (2007, p. 291). Los "otros" conforman una "totalidad", pues cada existencia encuentra su ser en otra existencia, aunque se trata de una "totalidad destotalizada, ya que al ser la existencia-para-otro rechazo radical del otro, ninguna síntesis totalitaria y unificadora de los 'otros' es posible" (2007, p. 291). Además del rechazo radical del otro, cuyas secuelas no tardaremos en descubrir, hay que tener en cuenta la contingencia original de todas los existentes y de sus mutuas relaciones para entender por qué, desde este sistema de pensamiento, la unidad absoluta entre los para-sí es tan inconcebible como irrealizable.

De lo dicho hasta acá se desprende que, en la medida en que se conciba al otro como objeto y la relación con él como una "relación de objetidad" (objectité), la existencia del otro para mí se manifestará inevitablemente como relación de "conocimiento a conocimiento" y, por lo tanto, su existencia no pasará de ser una mera conjetura. Sartre, por el contrario, afirma que mi relación fundamental con el otro es una "relación de ser a ser". Así, y solo así, descubro el ser que soy, a la par que el otro me es dado como sujeto, es decir, como presencia real: "en persona". Esto no quiere decir que al menos una de las modalidades en que el otro se me presenta sea la objetidad. En efecto, cuando veo a tal o cual mujer, a tal o cual hombre, a un ser humano particular en cualquier situación imaginable, lo que veo son "objetos". Pero la modalidad original, respecto de la cual la modalidad anterior es una versión degradada, es aquella en la que el otro se me revela como sujeto cierto, indubitable. Esta relación es la que tiene lugar a través de la mirada del otro. Cuando el otro me mira se revela, simultáneamente, mi ser-objeto para el otro y el ser-sujeto del otro para mí. Se entiende, entonces, que la relación original con el otro esté basada en la posibilidad siempre presente de "ser-visto-por-otro": "otro es, por principio, aquel que me mira" (2007, p. 297).

Cuando el otro me mira haciéndome sentir vergüenza, miedo u orgullo (tales son los ejemplos a los que recurre el argumento), no solo experimento mi objetidad, sino también la subjetividad del otro, es decir, su libertad. El otro aparece como "límite de mi libertad" (2007, p. 301), como "alienación de mis propias posibilidades" (2007, p. 302). Ante la mirada del otro, "ya no soy amo de la situación" (2007, p. 304). Sartre llega a afirmar que, en tanto $\mathrm{y}$ en cuanto dependo de una libertad que no es la mía, me aparezco al otro como "esclavo": "estoy en peligro. Y este peligro no es un accidente, sino la estructura permanente de mi ser-para-otro" (2007, p. 307). La descripción de la irrupción del otro está visiblemente cargada de connotaciones negativas cuyos efectos examinaré más adelante. De momento, me atengo a la conclusión a la que llega Sartre sobre esta cuestión: "El hecho del otro es incontestable y me alcanza en pleno corazón. Lo realizo a través del malestar; por él estoy perpetuamente en peligro" (2007, p. 314).

Ahora bien, el otro no es únicamente el sujeto que me mira, sino también el objeto que miro. En este sentido, la realidad-humana puede experimentarse como "ser-mirado" o como "serque-mira". La mirada, en una dirección o en otra, aliena a la realidad-humana de sus posibilidades 0 , más específicamente, de su trascendencia. Por esta razón, Sartre puede decir que el otro, en cuanto otro-objeto, me aparece como "trascendencia trascendida", y en cuanto otro-sujeto, me aparece como "trascendencia trascendente". Toda la cuestión del otro se encuentra determinada por la mirada, según quién sea visto y quién vea. Ambas posiciones tienen como condición necesaria que se den de manera alternada, nunca simultáneamente. En efecto, en un primer momento en el que mi posición es la del ser-mirado, el otro se me presenta como sujeto; pero en un segundo momento en el que me identifico con el ser-que-mira, el otro se me presenta como objeto. Sartre explica que la primera negación es motivo de la segunda. $\mathrm{El}$ hecho de que el otro, primeramente, limite mi libertad y me ponga "fuera de juego", funciona como motivación para que yo tome conciencia de mi subjetividad, hasta entonces rebajada, y de este modo invierta la situación. Un mismo ser no puede realizar ambas negaciones a la vez, de ahí que la aparición de una conlleve necesariamente el 
ocultamiento de la otra. Así, cada término - “yo" y "otro" - se constituye por negación recíproca y en interioridad. Esto quiere decir que cada uno de los términos está determinado en lo más profundo de su ser por un ser que no es él. El otro me define desde el interior. No puedo dudar de su existencia sin dudar al mismo tiempo de la mía.

Las condiciones formuladas más arriba para que una teoría sobre el otro pueda ser considerada válida encuentran aquí su cumplimiento lógico. Elucidada la estructura ontológica del "ser-para-otro" y suponiendo la validez de este razonamiento, todavía hay que preguntarse en qué medida esta teoría puede ser de utilidad para explicar la "experiencia del nosotros".

\section{Las actitudes hacia el otro y el "nosotros"}

Con la finalidad de avanzar en la comprensión de las relaciones concretas con el otro y del surgimiento del nosotros, es necesario referirse a una cuestión hasta ahora implícita en el argumento de Sartre. Mi relación con el otro, independientemente de quién mire y quién sea mirado, se manifiesta a través de los cuerpos. El encuentro con el otro está condicionado por la situación de los cuerpos en el mundo, es decir, por la facticidad y la contingencia irreductible que los cuerpos son. Las relaciones particulares de mi ser con otro ser suponen desde el comienzo que ambos existimos como cuerpos en el mundo, que somos, pues, "cuerpos-en-situación". En su definición mínima, el cuerpo es "la forma contingente que toma la necesidad de mi contingencia" (2007, p. 348). En sentido ontológico, el cuerpo no se diferencia del para-sí o, mejor aún, de la situación existencial del para-sí.

En este mismo sentido, el cuerpo replica la estructura del ser de la conciencia. El cuerpo aparece en el mundo según dos modos de ser: el cuerpo existe para sí y también existe para otro. El ser-para-sí y el ser-para-otro son, cada uno a su manera, cuerpo.

El ser-para-sí debe ser enteramente cuerpo y debe ser enteramente conciencia: no podría estar unido a un cuerpo. De igual modo, el ser-para-otro es enteramente cuerpo; no hay allí 'fenómenos psíquicos' que deban ser unidos al cuerpo; no hay nada detrás del cuerpo. Pero el cuerpo es enteramente 'psíquico'. (2007, pp. 344-345).

Con todo, la identificación entre el cuerpo, entendido como forma o materia de la psique, y el ser de la conciencia debe ser relativizada si de lo que se trata es de entender el orden estricto que sigue la ontología sartreana. De lo que se acaba de decir, no debe concluirse que la relación "original" o "fundamental" con el otro tiene lugar a través de los cuerpos. Si esto fuera así, se recaería en una "pura relación de exterioridad", cuando de lo que se trata justamente es de concebir la relación como "negación interna" (2007, p. 379). Para evitar semejante riesgo, Sartre reafirma una vez más el privilegio de la conciencia, esta vez frente a las posibilidades de los cuerpos:

\section{la aparición del cuerpo del otro no es el encuentro primero, por el contrario, no es más que un episodio de mis relaciones con el otro (...), o, si se quiere, el otro existe para mí primero y lo capto en su cuerpo después; el cuerpo del otro es para mí una estructura secundaria. (2007, p. 379).}

El mismo privilegio, la misma primacía de la conciencia y de la lógica que ella prescribe puede verificarse en el estudio sobre las relaciones concretas y particulares con el otro, ya se trate del deseo, del amor, del odio o de cualquier actitud derivada de estas. Dichas relaciones no son "simples especificaciones de la relación fundamental", pero "cada una incluye en sí misma la relación original con el otro como su estructura esencial y su fundamento" (2007, p. 401). Esto significa que el conjunto de mis actitudes hacia el otro - así como las del otro hacia mí - por singulares y diferentes que sean, se rigen por la lógica paradigmática de la mirada.

El otro me mira y a través de su mirada me objetiva hasta el punto de poseerme. Lo que el otro posee es nada menos que "el secreto de mi ser". Desde ese momento, "el sentido profundo de mi ser permanece fuera de mí" (2007, p. 403). Frente a esta situación se plantean entonces "dos actitudes opuestas" y "primitivas": o bien yo miro al otro, y de este modo lo objetivo, me libero de su dominio y a la vez lo domino, negando el ser que el otro me atribuye, o bien intento asimilarme la libertad del otro, que es fundamento de mi ser, pero dejando 
intacta la libertad, la alteridad del otro, a efectos de convertirme en mi propio fundamento.

Una actitud es motivación de la otra. Sartre afirma que no hay "dialéctica" de las dos actitudes hacia el otro sino "círculo": "(c)ada una de ellas es la muerte de la otra, es decir que el fracaso de una motiva la adopción de la otra" (2007, p. 403). El denominador común de las dos clases de actitudes que aquí se describen es precisamente el "fracaso" (échec). Esta palabra se repite como un mantra tanto en la caracterización de las conductas donde el para-sí intenta recuperar su ser asimilándose la libertad del otro (amor, lenguaje, masoquismo, etc.), como en la de aquellas conductas opuestas donde el para-sí adopta la posición del ser-quemira para enfrentar la libertad del otro mediante su propia libertad (indiferencia, deseo sexual, sadismo, odio, etc.). No hay salida ni alternativa a este círculo de permanentes fracasos. Aunque esencialmente diferentes, ambas tentativas, en el fondo, se malogran por la misma razón: "solo podríamos adoptar una actitud consistente hacia el otro si este nos fuera revelado a la vez como sujeto y como objeto, como trascendencia-trascendente y como trascendencia-trascendida, lo que en principio es imposible" (2007, p. 448).

Después de haber visto en la sección anterior la valoración eminentemente negativa de la irrupción del otro y, más aún, después de constatar la imposibilidad necesaria de cualquier clase de relación consistente o estable con el otro, no puede sorprender el lugar decisivo que ocupa el conflicto en esta teoría. Como afirma el propio Sartre justo antes de comenzar a examinar las distintas actitudes hacia el otro: "(1)as descripciones que siguen deben ser consideradas, pues, en la perspectiva del conflicto. El conflicto es el sentido original del ser-para-otro"8 (2007, p. 404).

He aquí una de las claves para comprender el significado, los alcances y las limitaciones de la así llamada "experiencia del nosotros". Justamente, lo que Sartre entiende por nosotros refiera a "ciertas experiencia concretas en las que nos descubrimos, no en conflicto con el otro, sino en comunidad con él" (2007, p. 453). Así lo expresa en el último apartado del Capítulo III de la Tercera parte, titulado "El 'ser-con' (Mitsein) y el "nosotros"'. Ahora bien, si el "conflicto es el sentido original del ser-para-otro", esto quiere decir que el nosotros o la "comunidad" —noción que para Sartre, al igual que para la inmensa mayoría de los pensadores sociales de los siglos XIX y XX equivale a "solidaridad" - , constituye una experiencia deficiente respecto del sentido esencial del para-otro. Y sin embargo es una experiencia efectiva y cotidiana de mi relación con los otros, ya no con tal o cual otro particular, sino con los otros en general. Por esta razón se puede decir que el "nosotros" representa para Sartre la experiencia más cercana a lo que habitualmente entendemos por realidad social.

No se habrá pasado por alto que la tesis y la suma de los ejemplos referidos hasta aquí involucran solamente dos conciencias: la mía y la del otro. La experiencia del nosotros, en cambio, desde que tiene lugar, compromete una pluralidad de conciencias. La existencia de una multiplicidad de individuos que mantienen relaciones entre sí es un hecho que Sartre no deja de reconocer, pero que al mismo tiempo tiene dificultades para explicar desde su matriz ontológica. Veamos cómo procede.

Sartre empieza por criticar el concepto de "ser-con", que Heidegger había definido como el modo esencial de ser o estar con los otros: una "relación de ser" que "ya está dada con el ser del Dasein" (Heidegger, 2016, p. 144). En lo que respecta a esta cuestión, lo que le critica al autor de Ser y tiempo es el hecho de no partir de la interioridad del cogito para desde allí encontrar el ser del otro. Esta es la objeción fundamental que le realiza a la concepción heideggeriana del Mitsein. En cualquier caso, esto no significa que la experiencia de sercon otros carezca de realidad. Esto significa, más bien, que el fundamento de esta experiencia hay que buscarlo en el ser-para-otro. "El ser-para-el otro precede y funda el ser-con-el otro" (2007, p. 455). De lo que se trata, una vez más, es de apelar a la conciencia. Ni a la conciencia intersubjetiva ni a la conciencia colectiva — ambas posibilidades quedaron descartadas desde el inicio-, sino a la conciencia individual. Esta es la única de la que no está permitido dudar y, en consecuencia, la única que puede experimentar un nosotros.

Sartre inscribe su estudio de la "experiencia del nosotros" bajo la lógica de la mirada. Esto le permite examinar fenómenos colectivos sin por ello abandonar el fundamento ontológico de su teoría. $\mathrm{Y}$ es así que distingue "dos formas radicalmente diferentes de la experiencia del nosotros y las dos formas corresponden exactamente al ser-que-mira y al ser-mirado, que constituyen las relaciones fundamentales del para-sí con el otro" (2007, p. 
455). Estas formas son el "nosotros-sujeto" y el "nosotros-objeto".

El nosotros-objeto es tratado en primer lugar. Este refiere a una situación donde se experimenta en comunidad con otro (u otros) la vergüenza y la alienación provocada por la mirada de un tercero. Las personas comprometidas en este "nosotros" hacen la experiencia común de aparecer como objetos ante la aparición de un tercero que los mira. La figura del tercero da lugar a numerosas posibilidades, dependiendo del "ser-mirada" de cada uno de los participantes de esta comunidad. De cualquier forma, el ejemplo del que se sirve Sartre para explicar las principales características del nosotros-objeto es lo suficientemente claro: me encuentro en un conflicto con otro, viene un tercero y nos mira; lo que sucede es que tanto yo como el otro con el que hasta entonces estaba enfrentado vivenciamos simultáneamente, aunque cada uno para sí mismo, la objetidad que nos impone el tercero. De esta suerte, "experimento la existencia, en el mundo del tercero, de una situación-forma objetiva donde, el otro y yo, figuramos a título de estructuras equivalentes y solidarias" (2007, p. 458). Lo que antes era conflicto con el otro ahora se transforma en una suerte de "nivelación" o "comunidad de equivalencia" donde ambos somos trascendidos por la subjetividad de un tercero. En este caso, lo que se vive como nosotros es una totalidad inexorable que cada uno de los seres trascendidos debemos asumir individualmente pero en solidaridad con el otro.

Las tres situaciones elegidas por Sartre para ejemplificar el nosotros-objeto están fuertemente enlazadas: el "trabajo en común", la "conciencia de clase" y la "psicología de las masas". Cuando bajo la mirada de un tercero un conjunto de personas trabaja sobre un mismo objeto, por ejemplo en una cadena de montaje, se experimenta una forma de nosotros singular, posibilitado por el ser-que-mira y por el objeto mismo que se produce en común. Los trabajadores, captados por la mirada de un otro y aprehendidos por la propia materialidad de aquello que producen, se experimentan como "una disposición instrumental y técnica de medios en la cual cada uno tiene su lugar asignado para un fin" (2007, pp. 459-460). En cuanto a la "conciencia de clase", la posición de Sartre resulta cuando menos polémica. Esta situación colectiva y altamente estructurada vale fundamentalmente para la "clase oprimida". La "clase opresora" cumple aquí el papel "de un tercero perpetuo que los considera y los trasciende por su libertad" (2007, p. 460). Según esta perspectiva, lo que constituye la conciencia de clase de los trabajadores no tiene ninguna relación con las condiciones sociales, económicas ni políticas de este colectivo. Lo verdaderamente decisivo, el "hecho primero" a decir de Sartre, "es que el miembro de la colectividad oprimida (...) capta su condición y la de los otros miembros de esta colectividad como mirada y pensada por conciencias que se le escapan" (2007, p. 461). Esto es, por la conciencia de un tercero que los trasciende perpetuamente, trátese del "amo", del "señor feudal" o del "capitalista". Por último, en cuanto a la "psicología de las masas", Sartre no innova. Repite el gesto de menosprecio característico en el tratamiento tradicional del problema de las masas, pero lo plantea en sus propios términos. El nosotros de la masa es experimentado por los individuos que la conforman como una forma de servidumbre voluntaria o, más exactamente, como una forma fracasada de amor que conduce al masoquismo: "la masa fue constituida como masa por la mirada del jefe o del orador; su unidad es una unidad-objeto que cada uno de sus miembros lee en la mirada del tercero que la domina" (2007, p. 462).

Lejos de lo que podría esperarse, el nosotrossujeto no es el doble invertido del nosotros-objeto. Sartre advierte desde el comienzo del apartado que se trata de dos experiencias completamente distintas, a tal punto que ni siquiera poseen el mismo estatuto ontológico. El nosotros-sujeto se experimenta como tal por un individuo que participa con otro (u otros) de un proyecto común pero a través del cual no se revela ningún objeto. Cada una de las personas que participa de esta comunidad lo hace a modo de trascendencia indeterminada, modo que Sartre identifica con el "se" (on) o realidad impersonal. No son sujetos en sentido estricto porque ello implicaría que se capten simultáneamente como subjetividades o trascendencias-trascendentes, lo cual, como ya se ha comprobado, desde esta perspectiva ontológica es imposible. De ahí que Sartre ponga en duda desde el principio la posibilidad de "un plural del "yo", que es precisamente lo que indica el pronombre "nosotros" en su acepción general.

No obstante, el nosotros-sujeto remite a una experiencia concreta y es preciso caracterizarla. Este se manifiesta, por ejemplo, en la comunidad 
episódica de pasajeros que hacen una misma combinación en el subterráneo. Es la construcción y la disposición material del subterráneo (mapas, escaleras, señalización, pasillos, etc.) la que me descubre mi trascendencia indiferenciada junto a la de muchos otros seres de los que apenas me distingo. En esta organización particular de cosas y cuerpos, "perdemos nuestra individualidad real, ya que el proyecto que somos es precisamente el proyecto que son los otros" (2007, p. 464). La coincidencia momentánea de mi proyecto con el proyecto de los otros en una situación en la que todos actuamos como una trascendencia cualquiera, posibilita "la experiencia de una trascendencia común y dirigida hacia un único objetivo" (2007, p. 465).

Dos indicaciones de Sartre terminan por aclarar la peculiaridad del nosotros-sujeto. Por una parte, la define como experiencia psicológica y subjetiva de una conciencia particular. En ningún caso se trata de una experiencia ontológica, ya que no se produce la "unificación real de los para-sí considerados", ni es la "prueba inmediata de su trascendencia en cuanto tal" (2007, p. 465). Basta con que un para-sí experimente el "ritmo común", repetitivo y regular con otros para-sí, por ejemplo en "la marcha cadenciosa de los soldados" o en el "trabajo rítmico de los equipos" (2007, p. 465). El ritmo y el fin perseguido por cada individuo se funden lateralmente con el ritmo y el fin colectivo. El nosotros-sujeto expresa un modo de sentirse con los otros pero no constituye una relación original con los otros. Simboliza una unidad irrealizable, puesto que "las subjetividades permanecen fuera de alcance y radicalmente separadas" (2007, p. 466). Por otra parte, y en sintonía con lo anterior, esta experiencia no es primera, sino segunda, en la medida en que presupone la existencia del otro. Es decir que el otro tiene que estar dado previamente para que el ser-con otros sea posible. Si un para-sí se realiza como trascendencia cualquiera, como el "se" que consume una mercancía o que acata una señal de tránsito, es porque otro (u otros) ya lo capta(n) de esta manera. En definitiva, lo que se busca mostrar a través de estas indicaciones es que "la experiencia del nosotros-sujeto no tiene ningún valor de revelación metafísica; depende estrechamente de las diferentes formas del paraotro y no es más que un enriquecimiento empírico de algunas de entre ellas" (2007, p. 468).
Sartre llega a la conclusión, por demás esperable, de que la experiencia del nosotros solo en apariencia es contradictoria con lo que se podría llamar la "ontología de la mirada" (Catalano, 2005, p. 19). A pesar de sus enormes diferencias, incluso a nivel ontológico, las dos formas del "nosotros" son manifestaciones derivadas de la experiencia original del ser-para-otro. En resumidas cuentas, ninguna de ellas constituye por sí misma una estructura ontológica.

\begin{abstract}
¿Se trata del nosotros-objeto? Es directamente dependiente del tercero, es decir de mi serpara-el otro y es sobre el fundamento de mi ser-afuera-para-el otro que se constituye. ¿Se trata del nosotros-sujeto? Es una experiencia psicológica que supone, de un modo u otro, que la existencia del otro en cuanto tal nos haya sido revelada. Sería en vano, entonces, que la realidad-humana buscara salir de este dilema: trascender al otro o dejarse trascender por él. La esencia de las relaciones entre conciencias no es el Mitsein, es el conflicto. ${ }^{10}$ (2007, p. 470).
\end{abstract}

\section{En el umbral de lo social}

¿Qué nos dice la experiencia del nosotros acerca de la vida en común en sentido amplio? ¿Qué implicaciones tiene esta vivencia singular donde se miden y compulsan el encuentro y el desencuentro de los existentes humanos para la comprensión del mundo social? Se dirá, tal vez, que en El ser y la nada Sartre no tenía por objetivo elaborar una teoría de lo social. Lo cual es innegable. El propósito que perseguía, por lo demás explicitado de entrada, era dar comienzo a una teoría del ser en general. Sin embargo, así como las teorías sociales comportan de manera explícita o implícita una determinada concepción del ser, del mismo modo, las ontologías, especialmente aquellas centradas en lo humano, conllevan una cierta comprensión de lo social.

Convengamos que en El ser y la nada el horizonte social se vuelve visible lenta y gradualmente. La figura del nosotros solo aparece al final del largo análisis consagrado al ser-para-otro. Sartre dedica la mayor parte de este tramo del libro a convalidar su tesis sobre la existencia del otro y a describir la relación original y las relaciones concretas entre los seres. Es al cabo de su investigación sobre el ser-para-otro donde deja de lado "el caso simple donde yo estoy solo frente al otro 
solo" para reconocer que "mi relación con el otro aparece sobre el fondo infinito de mi relación y de $s u$ relación con todos los otros" (2007, p. 456). El reconocimiento de ese fondo sin fondo, que no es nada distinto del trasfondo social donde toda vinculación humana encuentra lugar, puede leerse como un movimiento de apertura hacia un mundo donde no solo hay el otro, sino los otros; un mundo donde la relación con el otro es indisociable de las relaciones múltiples y heterogéneas entre los muchos existentes. Pero hasta dónde llega efectivamente este movimiento es difícil de determinar. Entre otras razones porque cuando se indagan las relaciones que involucran más de dos individuos no se tarda en descubrir que dichas relaciones se encuentran fuertemente condicionadas. A pesar de que en un primer momento de la exposición el nosotros es anunciado como "un concepto que subsume una infinita variedad de experiencias posibles" (2007, p. 453), las definiciones y los ejemplos de las dos formas del "nosotros" que siguen a continuación representan formas muy determinadas del ser-con otros. En efecto, se pueden imaginar innumerables situaciones que no se adecúan a los criterios del nosotros-objeto ni a los del nosotros-sujeto: desde situaciones particulares, como las que pueden darse en un grupo más o menos numeroso donde exista un mínimo de reciprocidad "positiva" o por lo menos no necesariamente "negativa" entre los participantes, hasta la situación límite y en extremo general como es el caso de la totalidad de la especie humana. Con respecto a esta última, Sartre es categórico. Cualquier proyección de un nosotros que abarque a la totalidad de la humanidad, ya sea en calidad de "objeto" o de "sujeto", no pasa de ser un ideal inalcanzable y destinado a fracasar. En el primer caso, porque solo a los ojos de un tercero no humano la suma de los hombres y las mujeres podría constituir un nosotros, lo cual implica la idea de Dios; y en el segundo caso por la imposibilidad, ya esgrimida, de que las subjetividades se capten mutuamente como tales en un todo unificado (2007, pp. 463, 469).

El lugar relegado y el estatuto ontológicamente degradado que Sartre le otorga a la experiencia del nosotros en modo alguno son hechos casuales. Muy por el contrario, son signos inequívocos del privilegio que detenta el cogito en su teoría. Este privilegio, reiterado constantemente a lo largo del libro, no es un impedimento para que Sartre reflexione sobre el carácter social de la existencia, pero sin duda constituye una limitación considerable. Lo que afirmo es que el mayor impedimento en este sentido viene dado con y desde el "punto de partida". El esquema dualista de la mirada está proyectado hasta en los más mínimos detalles a la medida de una ontología fenomenológica fundada en el cogito. Recordemos que este devela no solo la verdad de mi existencia. En la versión ampliada de Sartre, también devela la verdad de la existencia del otro y la de mi existencia para otro. Al interior del esquema ser-mirado / ser-que-mira, la explicación de las relaciones diádicas preserva una coherencia lógica incontestable, independientemente de cómo se juzgue la interpretación sartreana de la realidad-humana. Lo cierto es que desde el momento en que se descubre el "fondo infinito" de las relaciones donde aparecen implicados tres o más conciencias, la lógica tambalea. Esta solo se mantiene en pie - si es que lo hace- al precio de un razonamiento tan forzado como forzoso que deja al desnudo las debilidades de este modelo teórico para pensar la realidad social en toda su complejidad.

Sucede que la mirada es siempre la mirada de un ser singular, pudiendo ser la mía, la del otro o la del tercero. Sartre se encarga de dejar en claro que la mirada del otro-sujeto "no se da bajo forma de pluralidad" (2007, p. 320). La pluralidad pertenece a los objetos, no a los sujetos. Ciertamente puedo ser mirado por el otro. También puedo ser mirado junto al otro por un tercero, como en la situación que da lugar al nosotros-objeto. Pero no hay mirada del "nosotros" sujeto, solo hay mirada del "yo". En aquellas situaciones públicas en las que se actúa en presencia de otros, al "interpretar un papel o dar una conferencia”, según los ejemplos de Sartre, los otros no cuentan como sujetos, sino que se presentan como "realidad impalpable, fugaz y omnipresente" (2007, p. 321), como el "se" impersonal de las individualidades que forman el nosotros-sujeto. La mirada en presencia de la cual se actúa no puede ser atribuida a "la clase" o "el auditorio": "no tenemos conciencia, en efecto, de un ser concreto e individualizado con una conciencia colectiva; estas son imágenes que luego podrán servir para traducir nuestra experiencia y que la traicionarán más que a medias" (2007, p. 321).

$\mathrm{Al}$ introducir la experiencia del nosotros y, a través de ella, la oportunidad de ir más allá de las relaciones diádicas y esencialmente conflictivas, Sartre vislumbra un mundo de infinitas 
posibilidades relacionales. Pero a decir verdad no hace más que vislumbrarlo. Fiel al principio rector de su filosofía, siempre termina por replegarse sobre la conciencia del individuo. En su concepción del mundo no hay lugar para una conciencia intersubjetiva ni para una conciencia colectiva, dado que "la relación o la totalidad" están "desprovistas de autonomía ontológica por fuera de la actividad constituyente del para-sí" (Tertulian, 1990, p. 403). La plena autonomía de la conciencia individual tiene consecuencias tanto en el plano ontológico como en el plano óntico de las relaciones concretas con el otro. Como hemos viso, el nosotros no es más que una derivación posible de la experiencia original del ser para-sí-para-otro de la realidadhumana. Aunque, por cierto, el nosotros-sujeto y el nosotros-objeto no tienen el mismo rango ontológico. El primero es una experiencia puramente psicológica sin relevancia metafísica. Mientras que el segundo, en cambio, es la unión efectiva de dos (o más) conciencias que se experimentan simultáneamente como objetos por la mirada de un tercero que a la vez se experimenta como sujeto. Sin embargo, el nosotros-objeto tampoco es una estructura ontológica autónoma e independiente. Este depende y se funda en el tercero, es decir, en un ser perfectamente individual. La figura del tercero no es suficiente para descubrir una dimensión propiamente social en esta teoría. Como señala Flynn (1984), el tercero actúa como sujeto individual, no como sujeto social. La solidaridad experimentada en el nosotros-objeto es constituida por el tercero. Asimismo, este impone la alienación al "nosotros" tal como un individuo impone la objetividad a otro "yo", transfigurando el dualismo inherente al fenómeno de la mirada en una supuesta relación entre tres o más personas. En este sentido, "está justificada la caracterización de las relaciones triádicas en El ser y la nada como verdaderas díadas encubiertas y la interpretación del Tercero introducida en este libro como el Otro en general" (Flynn, 1984, p. 26). La exclusión deliberada de una mirada plural reduce a su mínima expresión la profusión de cuerpos, posiciones y disposiciones, fuerzas y diferencias, modos de hacer y pensar que se entrecruzan en el seno de la vida social.

Incluso si dejamos de lado el reduccionismo psicológico operado por Sartre en el nosotrossujeto y nos aferramos a la aparición del tercero en la experiencia del nosotros-objeto, desde el punto de vista óntico o empírico la teoría se muestra incapaz para dar cuenta de la pluralidad irreductible de relaciones que configuran la trama social. Aquello de lo aquí no se da cuenta, por fuerza de lógica, es acaso el rasgo distintivo de la existencia en común, a saber, la infinita variedad y variabilidad de relaciones de las que participan los existentes asociados o disociados, de manera directa o indirecta, en un momento dado de la historia, en condiciones sociales, económicas y políticas determinadas.

En efecto, Sartre vislumbra un mundo en el que no llega a adentrarse. Lo ve a la distancia y en alguna medida alcanza a reconocerlo. Pero es sabido que allí, justamente, no penetra la mirada. De esta manera, se puede afirmar que en $\mathrm{El} \mathrm{ser}$ y la nada Sartre permanece en el umbral de lo social. Se mantiene sobre el límite que le impone su propia lógica de pensamiento. Los supuestos filosóficos de su ontología fenomenológica no le permiten ir más lejos sin tener que enfrentarse a serias contradicciones que amenazan desde sus bases la arquitectónica de su construcción teórica. No obstante, es justo decir que paralelamente a su sinuoso acercamiento al marxismo a partir de mediados de la década de 1940, Sartre comienza a identificar y afrontar algunas de estas contradicciones. Al menos aquellas que suponen una limitación mayor para poder pensar, con amplitud de miras, el carácter eminentemente social de la existencia humana. La confluencia entre los legados del "marxismo" y el "existencialismo" - ambos resignificados por Sartre a los efectos de una coexistencia posible- desembocará en ese libro monumental y difícilmente catalogable que es la Crítica de la Razón dialéctica. Los nuevos conceptos adaptados al análisis social que introduce esta obra (la praxis individual y colectiva, lo práctico-inerte, la serie, el grupo, la clase y el tercero regulador, entre tantos otros que se podrían mencionar) han de ser interpretados a la luz de los valiosos descubrimientos de El ser y la nada, pero también y sobre todo a la luz de lo que en este primer ensayo ontológico de Sartre representa un umbral o un límite aparentemente infranqueable. Si la inquietud por la intrincación entre ontología y teoría social pone al descubierto una vinculación poco trabajada y que por eso mismo amerita ser desarrollada, entonces es esperable que otras investigaciones avancen sobre lo conocido y lo desconocido de esta inmensa cuestión. 


\section{Referencias}

Alvaro, D. (2015). El problema de la comunidad. Marx, Tönnies, Weber. Buenos Aires: Prometeo.

Aron, R. (1969). Marxism and the Existentialists. New York: Harper and Row.

Aron, R. (1973). Histoire et dialectique de la violence. Paris: Gallimard.

Boileau, K. C. (2000). Genuine reciprocity and group authenticity. Foucault's developments or Sartre's social ontology. Lanham, Md.: University Press of America.

Catalano, J. S. (2005). Sartre's Ontology from Being and Nothingness to The Family Idiot. Sartre Studies International, 11(1-2), 17-30.

Flynn, T. R. (1984). Sartre and Marxist Existentialism: The Test Case of Collective Responsibility. Chicago: University of Chicago Press.

Flynn, T. R. (2014). Sartre: A Philosophical Biography. Cambridge: Cambridge University Press.

Goldmann, L. (1970). Marxisme et sciences humaines. Paris: Gallimard.

Heidegger, M. (2016). Ser y tiempo. Madrid: Trotta.
Lukács, G. (1984). Ontologie des gesellschaftlichen Seins, Werke, Band 13. Darmstadt-Neuwied: Luchterhand.

Lukács, G. (1986). Ontologie des gesellschaftlichen Seins, Werke, Band 14. Darmstadt-Neuwied: Luchterhand.

Merleau-Ponty, M. (2000), Les aventures de la dialectique. Paris: Gallimard.

Sartre, J.-P. (1966). El ser y la nada. Buenos Aires: Losada. Sartre, J.-P. (1984). El ser y la nada. Madrid: Alianza.

Sartre, J.-P. (1985). Critique de la raison dialectique. Tome I. Théorie des ensembles pratiques, précédé de Questions de méthode. Paris: Gallimard.

Sartre, J.-P. (2003). L'existentialisme est un humanisme. Paris: Gallimard.

Sartre, J.-P. (2007). L'être et le néant. Essai d'ontologie phénoménologique. Paris: Gallimard.

Tertulian, N. (1990). Entre Heidegger et Marx. Les Temps Modernes, $n^{\circ}$ spécial "Témoins de Sartre", 1, 398-412.

Theunissen, M. (2013). El otro. Estudios sobre ontología social contemporánea. México: FCE. 


\section{Notas}

$1 \quad$ El texto de la conferencia puede leerse de principio a fin como una defensa del existencialismo, pero es sobre todo en la "Discusión" que sigue a la conferencia donde se expone con total claridad y crudeza tanto la posición de Sartre como la de algunos de sus críticos de ese entonces. Véase Sartre (2003, pp. 79-109).

2 A partir de 1946, Sartre publica numerosos y variados textos que atestiguan su aproximación al marxismo. Probablemente, Cuestiones de método sea el que mejor resume su posición filosófico-política al momento de publicar la Crítica de la Razón dialéctica, en especial su primera parte, "Marxismo y existencialismo" (1985, pp. 16-39).

3 En este artículo me ocupo principalmente de El ser y la nada y solo trato de manera circunstancial otros textos de Sartre. Tampoco trato aquí los aspectos contextuales de su producción. Para un abordaje integral de su vida y sus obras reenvío a la reciente biografía filosófica de T. $\mathrm{R}$. Flynn (2014)

4 Téngase en cuenta que Sartre distingue cuidadosamente el cogito reflexivo o cartesiano del cogito prerreflexivo. Este último es el modo originario de la conciencia y, por lo tanto, es anterior a todo conocimiento. Con todo, no se trata aquí de dos conciencias, sino de dos formas interrelacionadas de la misma conciencia. A fin de subrayar el primado del ser sobre el conocer, que es un precepto básico del existencialismo y, a la vez, es lo que diferencia a esta filosofía de otras filosofías de la conciencia, Sartre postula que "es la conciencia no-reflexiva la que hace posible la reflexión: hay un cogito prerreflexivo que es la condición del cogito cartesiano" (2007, p. 19).

5 Sobre esta cuestión existe un amplio consenso entre los intérpretes. La mayoría de ellos, tanto los que asumen una posición crítica respecto del existencialismo sartreano como aquellos que parecen más próximos a este, reconocen la primacía ontológica del individuo sobre la colectividad en El ser y la nada. De este parecer son M. Merleau-Ponty (2000), M. Theunissen (2013), R. Aron (1969, 1973), L. Goldmann (1970), G. Lukács $(1984,1986)$ y T. R. Flynn (1984), por solo nombrar a algunos de los más conocidos.

6 La palabra autrui significa "otro", "el otro", "los otros" o "los demás", es decir, alguien que no soy "yo". También significa "prójimo", término usado frecuentemente para traducir la palabra francesa en las dos versiones castellanas más consultadas de El ser y la nada (Sartre, 1966, 1984). Aquí y en lo que sigue traduzco autrui por "otro" y en algunas ocasiones por "el otro", pues considero que son las acepciones más próximas al sentido general de esta noción en El ser y la nada. De hecho, en algunos pasajes especialmente importantes para este análisis, autrui se utiliza con un sentido contrario tanto a la idea de una pluralidad de individuos como a la idea de prójimo, en el sentido moral del término. A esto hay que agregar que, muy a menudo, Sartre combina las expresiones autrui, autre y l'autre, usándolas alternativamente como sinónimos.

7 La unicidad y la simultaneidad de este "surgimiento" son relativizadas por el propio Sartre en varios pasajes de su libro, instalando la duda sobre el hecho de que estos dos modos de existencia de la realidad-humana tengan efectivamente la misma prioridad ontológica. Por ejemplo, cuando afirma: "estas dos estructuras son inseparables. Pero al mismo tiempo, necesito del otro para captar en pleno todas las estructuras de mi ser, el para-sí reenvía [énfasis agregado] al para-otro" (2007, pp. 260-261). O también cuando afirma:

hay que pedirle al para-sí que nos entregue el paraotro, hay que pedirle a la inmanencia absoluta que nos lance en la trascendencia absoluta: en lo más profundo de mí mismo debo encontrar, no razones para creer en el otro, sino al otro mismo como no siendo yo. (2007, pp. 290-291).

8 La perspectiva elegida para dar cuenta de la relación con el otro habilitó una interpretación que le atribuye a la teoría de Sartre "un tinte particularmente hobbesiano", aduciendo que "las razones ofrecidas en El ser y la nada para explicar la naturaleza conflictiva de las relaciones interpersonales son ontológicas, ni históricas ni socioeconómicas" (Flynn, 1984 , p. 20). A propósito del pesimismo social del Sartre temprano, véase Boileau (2000).

9 Para una crítica del histórico privilegio acordado al concepto moderno de "comunidad", entendido como modelo social de relaciones positivas o solidarias, véase Alvaro (2015).

10 Llamo la atención sobre un hecho en el que no puedo detenerme pero que tampoco quisiera simplemente pasar por alto: aquí, como en otros pasajes del libro, Sartre equipara el Mitsein heideggeriano con una estructura solidaria, con un "vínculo solidarizante" (2007, p. 286). En principio, basta con leer el $\S 26$ de Ser y tiempo para percatarse del error —intencional o no- de la interpretación sartreana sobre esta cuestión. 